\title{
Morphological Feature Extraction for Automatic Registration of Multispectral Images
}

\author{
Antonio Plaza ${ }^{1}$, Jacqueline Le Moigne ${ }^{2}$, and Nathan S. Netanyahu ${ }^{3,4}$ \\ ${ }^{1}$ Department of Computer Science, University of Extremadura, E-10071, Cáceres, Spain \\ ${ }^{2}$ Advanced Architectures and Automation Branch, NASA Goddard Space Flight Center, Greenbelt, MD 20771 \\ ${ }^{3}$ Department of Computer Science, Bar-Ilan University, Ramat-Gan 52900, Israel \\ ${ }^{4}$ Center for Automation Research, University of Maryland, College Park, MD 20742
}

\begin{abstract}
The task of image registration can be divided into two major components, i.e., the extraction of control points or features from images, and the search among the extracted features for the matching pairs that represent the same feature in the images to be matched. Manual extraction of control features can be subjective and extremely time consuming, and often results in few usable points. On the other hand, automated feature extraction allows using invariant target features such as edges, corners, and line intersections as relevant landmarks for registration purposes. In this paper, we present an extension of a recently developed morphological approach for automatic extraction of landmark chips and corresponding windows in a fully unsupervised manner for the registration of multispectral images. Once a set of chip-window pairs is obtained, a (hierarchical) robust feature matching procedure, based on a multiresolution overcomplete wavelet decomposition scheme, is used for registration purposes. The proposed method is validated on a pair of remotely sensed scenes acquired by the Advanced Land Imager (ALI) multispectral instrument and the Hyperion hyperspectral instrument aboard NASA's Earth Observing-1 satellite.
\end{abstract}

\section{INTRODUCTION}

Many current and future applications in Earth Science, Space Science, and Exploration Science (will) require integration of data acquired from multiple sources. This type of integration provides necessary information for intelligent navigation and decision support systems in many real-time applications. Depending on the application at hand, the integration may be performed on-board or on-the-ground. In the case of Earth Science, for example, multiple data sources are due to satellite, aircraft, and ground measurement systems, where the data represent multiple temporal, spectral, and spatial resolutions. For the multisource data integration described above, a fast, automatic, reliable, and accurate image registration is essential.

Assuming that image data have been systematically corrected through prior navigation, image registration (or "precision correction") corresponds to feature-based matching of the image data, which refines georegistration to subpixel accuracy. Image registration is thus defined as the process that determines the best match between two or more images acquired at the same time or later, and wigth the same sensor of a different one. One dataset is regarded as the reference data and all other data, called input data (or sensed data), are matched relative to the reference data. The general process of image registration includes three main steps:

1) Extraction of features to be used in the matching process;

2) application of a feature matching strategy subject to a specific metric;

3) resampling of the data based on the correspondence computed from matched features.

A large number of automatic image registration methods have been proposed and surveys can be found in [1], [2]. In particular, previous work has focused on the comparison of different choices for Steps 1,2 above, where either an entire scene was registered (against a reference scene), or where several (small) chips, extracted typically around characteristic image features or landmarks from the reference scene, were registered initially against corresponding windows from the input scene [3]. The working assumption was that, once a registration system became operational, a database of such landmark chips would be available for use. Developing the capability of extracting these chips automatically and independently of any database presents the following relevant advantages:

- Elimination of a chip database that needs to be maintained regularly and adapted to each sensor;

- extracting automatically chip/windows that contain a very small amount of clouds, provided that preprocessing (of reference and input data) includes cloud detection;

- processing images of any size;

- reducing the initial registration error by extracting simultaneously chips (from the reference image) and corresponding windows (from the input image), thereby enhancing feature matching (or any optimization technique), as far as accuracy and speed are concerned; given that the initial conditions are closer to the parameters of the final transformation, these techniques serve as "refinement methods."

In this paper, we expand a recently proposed morphological method for automatic extraction of reference chips and corresponding input windows from remotely sensed image data [4]. Here, this method is adapted to the specific case of automatic registration of multispectral datasets. The paper is organized as 
follows. Section II outlines a previously developed morphological method which performs automated feature extraction for registration purposes. Section III addresses the modifications introduced in the morphological extraction algorithm in order to adapt it to processing of multispectral data sets. Section IV provides experimental results to validate the proposed approach. Finally, Section V concludes the paper with some remarks and hints at plausible future research.

\section{Morphological Feature Extraction}

Mathematical morphology [5] is a nonlinear image processing technique that was originally established by introducing fundamental operators applied to two sets. One set is processed by another set with certain spatial properties (i.e., shape and size), known as structuring element, which is translated over the spatial domain of the image. The structuring element acts as a filter for extracting or suppressing specific image structures. Following standard notation [5], let us consider a grayscale image $f$, defined on the discrete space $Z^{2}$, and a structuring element designed by $B \in Z^{2}$. The latter is usually 'flat' in the sense that it is defined in the spatial domain of the image (the $x-y$ plane). The morphological erosion of $f$ by $B$ is defined as

$$
(f \ominus B)(x, y)=\operatorname{Min}_{(s, t) \in Z^{2}(B)} f(x+s, y+t),
$$

where $(x, y) \in Z^{2}$ and $Z^{2}(B)$ denotes the set of discrete spatial coordinates associated with pixels lying within the neighborhood defined by $B$. In contrast, the morphological dilation of $f$ by $B$ is defined by

$$
(f \oplus B)(x, y)=\operatorname{Max}_{(s, t) \in Z^{2}(B)} f(x-s, y-t) .
$$

Using the same notation above, morphological opening and closing filters [5] can be respectively defined by:

$$
\begin{aligned}
& (f \circ B)(x, y)=((f \ominus B) \oplus B)(x, y), \\
& (f \bullet B)(x, y)=((f \oplus B) \ominus B)(x, y) .
\end{aligned}
$$

The two operators above have been used successfully to build morphological profiles by using combinations of structuring elements of progressively increased size [6]. However, these profiles cannot capture the directional information which is crucial to extract features such as edges, corners, and line intersections. To address this issue, we resort to so-called scale-orientation morphological profiles (SOMPs).

In order to mathematically define the concept of SOMPs, we first denote by $B_{p,(d x, d y)}$ a line segment structuring element with $p$ pixels along the line (in this work, we set $p=5$ ) and slope $d y / d x$. Using these notations, we can define the SOMPs by opening at a given pixel $(x, y)$ of an image $f$ as

$$
D_{(d x, d y)}^{\circ}(x, y)=\left\{\left[\left(f \circ B_{p,(d x, d y)}\right) \bullet B_{p,(d x, d y)}\right](x, y)\right\},
$$

where $p=\{1,2, \cdots, k\}$ and $k$ is the maximum number of considered scales. In similar terms, we can define the SOMPs by closing at $f(x, y)$ as follows:

$$
D_{(d x, d y)}^{\bullet}(x, y)=\left\{\left[\left(f \bullet B_{p,(d x, d y)}\right) \circ B_{p,(d x, d y)}\right](x, y)\right\} .
$$

In both cases, a measure of line strength can be computed for each scale and orientation by calculating a point-wise distance dist between the pixel $f(x, y)$ in the original image and the pixel at the same location in the image filtered by the considered line segment structuring element as follows:

$\mathbf{s}^{\circ}(x, y)=\left\{\operatorname{dist}\left(f(x, y),\left[\left(f \circ B_{p,(d x, d y)}\right) \bullet B_{p,(d x, d y)}\right](x, y)\right)\right\}$
$\mathbf{s}^{\bullet}(x, y)=\left\{\operatorname{dist}\left(f(x, y),\left[\left(f \bullet B_{p,(d x, d y)}\right) \circ B_{p,(d x, d y)}\right](x, y)\right)\right\}$

The resulting values from Eqs. (7) and (8) are combined into a feature vector $D(x, y)=\mathbf{s}^{\circ}(x, y) \cup f(x, y) \cup s^{\circ}(x, y)$ with dimensionality $L=2 k \times m$, where $k$ is the number of scales and $m$ is the number of orientations.

In order to automatically extract significant points of interest for registration, we use the concept of self-information [7]. (The more irregular the SOMP associated with a certain pixel, the higher the chance that it represents a corner pixel of an object.) Let the $L$ components of $D(x, y)$ be denoted by $\left\{d_{l}(x, y)\right\}_{l=1}^{L}$; then the self-information provided by the $l$ th component $(1 \leq l \leq L)$, can be defined by $I_{l}(x, y)=$ $-\log p_{l}(x, y)$, where

$$
p_{l}(x, y)=\frac{d_{l}(x, y)}{\sum_{l=1}^{L} d_{l}(x, y)} .
$$

Using the above definitions, the entropy of $D(x, y)$ can be obtained from

$$
H(D(x, y))=-\sum_{l=1}^{L} p_{l}(x, y) \times \log p_{l}(x, y) .
$$

\section{EXTENSION TO MULTi/HyPERSPECTRAL IMAGES}

The SOMP operators described in Section II have been used in previous work to automatically extract a set of landmark chips from a reference scene, and to find corresponding windows in the input scene in a fully unsupervised manner [4]. A measure of spectral information divergence (SID) [7] was first used to establish a preliminary matching of SOMP-based profiles in the input scene with those in the reference scene, followed by a (hierarchical) robust feature matching (RFM) procedure that makes use of a multiresolution overcomplete wavelet decomposition scheme to extract chip and window features at the various levels of the decomposition [8].

Although the proposed approach was successful for the registration of single spectral bands extracted from Landsat data sets [4], the following important aspects, which are crucial for the registration of multi/hyperspectral image datasets, were not addresed:

- Our previous approach did not incorporate any cloud detection procedure and the registration process was strongly affected by atmospheric interferers such as clouds and their shadows. In order to address this issue, we have resorted to spectral unmixing techniques [7] to automatically detect clouds (and their shadows) from the input scenes. Specifically, we considered the full spectral information in the data and extracted the brightest 
pixel vector using the automatic target generation process (ATGP) described in [7], [9]. The second iteration of ATGP provides the pixel which is most different, in an orthogonal sense, from the brightest pixel (essentially, the darkest pixel in the scene). These pixels (often associated with clouds and (cloud) shadows) are used to mask out cloudy/shaded areas by unmixing the scenes using linear unmixing [9] and removing pixels with very high abundance ( $90 \%$ or more) of either cloud or shadow prior to registration.

- If the pair of images to be registered are rotated against each other, as is indeed the case with ALI-Hyperion pairs, comparing the SOMPs directly (by using, e.g., a measure of spectral similarity) does not make sense, as opposed to the registration of Landsat data, for which a trivial rotation close to 0 degrees is expected. In fact, the SOMPs can be seen as histograms that indicate the degree of variation at different scales and orientations (see [6] for a more detailed explanation). Thus, if we calculate such a histogram for a certain pixel, then rotate the image, and recalculate the histogram, the order of the histogram bins (which show a measure of change of the pixel with regards to surrounding features in the scene at different scales and orientations) would be different, even though they refer to the same image pixel. To address this issue, we simply order the bins in the histogram in descending order (i.e., the lowest measures of change are placed first and the higher measures of change are placed last in the histogram) prior to spectral similarity matching using the SID measure. This issue is further discussed in [6].

Taking in mind the issues above, the sequence of steps implemented by our revised SOMP-based algorithm is:

1) Remove clouds and shadows in both the reference and input multispectral scenes using spectral unmixing.

2) Obtain the SOMPs for each pixel in the input scene, denoted by $D_{g}(x, y)$, and order the bins in descending order for each calculated SOMP.

3) For each pixel $f(x, y)$ in the reference scene, construct its SOMP denoted by $D_{f}(x, y)$ and calculate its entropy $H\left(D_{f}(x, y)\right)$.

4) Select the pixel $f\left(x^{\prime}, y^{\prime}\right)$ with the highest $H\left(D_{f}(x, y)\right)$ score (i.e., with the maximum entropy) in the reference scene and extract a reference chip centered on $f\left(x^{\prime}, y^{\prime}\right)$, where the chip size is defined by an input parameter.

5) Order the bins of the histogram given by $D_{f}\left(x^{\prime}, y^{\prime}\right)$ in descending order and compute the SID between $D_{f}\left(x^{\prime}, y^{\prime}\right)$ and all the SOMPs in the input scene obtained in Step 2, retaining the pixel coordinates $\left(x^{\prime \prime}, y^{\prime \prime}\right)$ of the pixel in the input scene for which the SID value is the smallest.

6) Extract a window centered on pixel $\left(x^{\prime \prime}, y^{\prime \prime}\right)$ in the input scene, where the window size is defined by an input parameter.

7) Return to Step 4 until a predefined number of different chip-window pairs is extracted.
TABLE I

ALI-HYPERION SPECTRAL BAND CORRESPONDENCES.

\begin{tabular}{c|c||c|c}
\hline \hline \multicolumn{2}{|c||}{ ALI } & \multicolumn{2}{c}{ Hyperion } \\
\hline $\begin{array}{c}\text { Band } \\
\text { number }\end{array}$ & $\begin{array}{c}\text { Spectral } \\
\text { range }(\mu \mathrm{m})\end{array}$ & $\begin{array}{c}\text { Band } \\
\text { number }\end{array}$ & $\begin{array}{c}\text { Average } \\
\text { wavelength }(\mathrm{nm})\end{array}$ \\
\hline 1 & $0.433-0.453$ & 9 & 436.99 \\
2 & $0.45-0.515$ & 14 & 487.87 \\
3 & $0.525-0.605$ & 22 & 569.27 \\
4 & $0.63-0.69$ & 31 & 660.85 \\
5 & $0.775-0.805$ & 44 & 793.13 \\
6 & $0.845-0.89$ & 50 & 854.18 \\
7 & $1.2-1.3$ & 106 & 1205.07 \\
8 & $1.55-1.75$ & 149 & 1638.81 \\
9 & $2.08-2.35$ & 207 & 2224.03 \\
\hline \hline
\end{tabular}

TABLE II

REGISTRATION RESULTS OBTAINED FOR FIVE CHIP-WINDOW PAIRS FROM A PAIR OF ALI-HYPERION MULTI/HYPERSPECTRAL IMAGES.

\begin{tabular}{ccccc}
\hline \hline $\begin{array}{c}\text { Chip-window } \\
\text { pair }\end{array}$ & $\begin{array}{c}\text { Size } \\
\text { (pixels) }\end{array}$ & $\begin{array}{c}\text { Rotation } \\
\text { (degrees) }\end{array}$ & $\begin{array}{c}\text { Initial shift } \\
t_{x}\end{array}$ & $\begin{array}{c}\text { Initial shift } \\
t_{y}\end{array}$ \\
\hline 1 & $128 \times 128$ & 11.28 & -1.442 & 0.153 \\
2 & $96 \times 96$ & 10.92 & 1.390 & 0.380 \\
3 & $128 \times 128$ & 10.84 & -0.020 & -0.70 \\
4 & $128 \times 128$ & 10.92 & 0.370 & -1.11 \\
5 & $128 \times 128$ & 10.69 & -0.720 & 0.04 \\
\hline \hline
\end{tabular}

\section{EXPERIMENTAL RESULTS}

We have tested the fully automated system described in section III using a pair of remotely sensed scenes collected by the Advanced Land Imager (ALI) multispectral instrument and the Hyperion hyperspectral instrument aboard NASA's Earth Observing-1 satellite. Although the instruments are mounted on the same platform, their spectral resolutions are different. For illustrative purposes, Table I shows the correspondences established between the 9 spectral bands provided by ALI and the corresponding Hyperion bands. A detailed description of band coverage and correspondences for ALI-Hyperion is available online (from http://eo1.usgs.gov/Hyperion Spectral_Coverage.htm).

In this study, we have selected a single pair of spectral bands from the considered ALI-Hyperion scenes for valiclation. The selected pair, displayed in Fig. 1, consists of ALl's band 7 and Hyperion's band 106. Both images are of size $256 \times$ 3352 pixels. Strong atmospheric interferers (i.e., clouds and shadows) can be observed in these ALI and Hyperion images depicted in the figure.

The automatic algorithm described in Section III was applied to the above scenes (with $k=5$ scales and $m=8$ orientations) to obtain a set of five chip-window pairs displayed in Fig. 2. Due to the narrow width of the ALI-Hyperion scenes (only 256 pixels), we had to carefully adjust the desired size of the extracted chip-window pairs. For all chip-window pairs, the size was set to $128 \times 128$ pixels, except for pair 2 (in Fig. 2), whose size was set to $96 \times 96$ pixels. In this particular case, the proximity to the image border prevented us from extracting a $128 \times 128$-pixel window around the image feature provided by the morphological operators. As can be observed from Fig. 2 , all selected chip-window pairs are free from clouds/shadows.

After applying the hierarchical version of the RFM tech- 


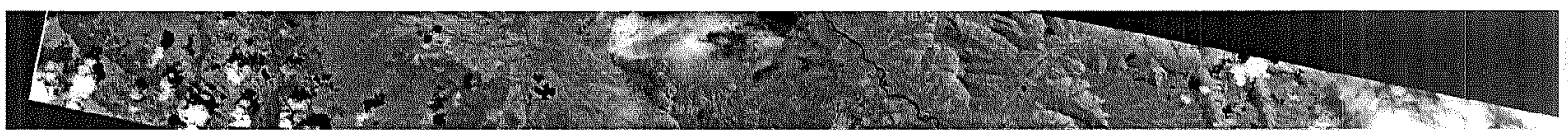

(a)

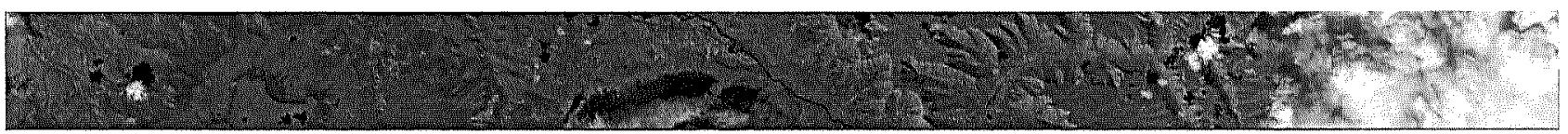

(b)

Fig. 1. Pair of corresponding ALI and Hyperion spectral bands used in our experiments. Both scenes are rotated 90 degrees (clockwise) for visualization purposes: (a) Band 7 (spectral range of $1.2-1.3 \mu \mathrm{m}$ ) of a multispectral image collected by ALI and (b) band 106 (average wavelength of $1205.07 \mathrm{~nm}$ ) of a hyperspectral image collected by Hyperion.

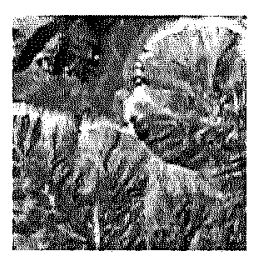

ALI chip 1

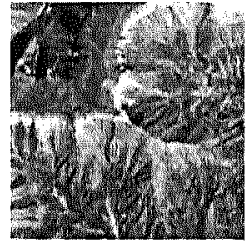

Hyperion window 1

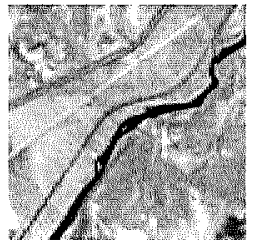

ALI chip 2

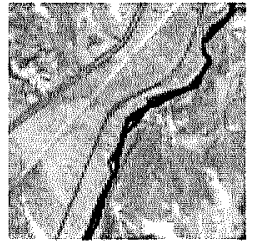

Hyperion window 2

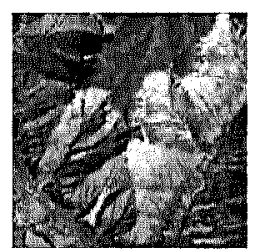

ALI chip 3

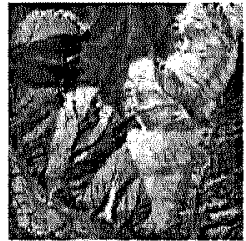

Hyperion window 3

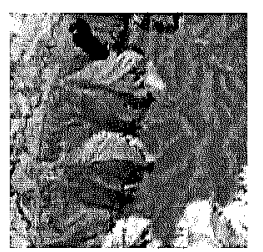

ALI chip 4

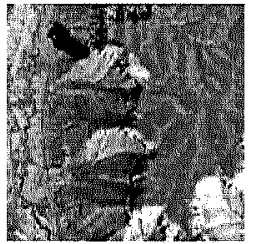

Hyperion window 4

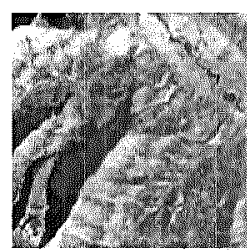

ALI chip 5

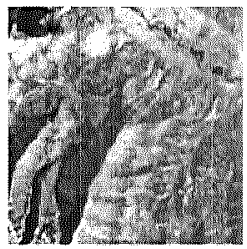

Hyperion window 5

Fig. 2. ALI chips (first row) and corresponding Hyperion windows (second row) extracted automatically by the proposed SOMP-based method. The size of chip-window pairs labeled as $1,3,4$, and 5 is $128 \times 128$ pixels, while the size of chip-window pair 2 is $96 \times 96$ pixels.

nique of [8], we obtained the individual chip-window registrations given in Table II. From these transformations, we used a robust estimation algorithm to derive a global transformation of 11.48 degrees $\left(t_{x}=-160.57\right.$ and $\left.t_{y}=152.41\right)$, which is in good agreement with a previously obtained result using a manual procedure, which resulted in a global transformation of 11.34 degrees $\left(t_{x}=-160.28\right.$ and $\left.t_{y}=149.8\right)$. This indicates that the proposed automatic registration approach can produce accurate results without prior knowledge.

\section{Conclusions and Future Research}

In this paper, we have described a fully automated system for registration of multispectral image datasets. The system is composed of two stages. First, a morphological feature extraction technique is used to obtain scale-orientation morphological profiles (SOMPs) centered on visually significant landmarks such as edges, corners, and line intersections. These profiles are then used to automatically extract a set of initially matched chip-window pairs which are then refined using a robust feature matching (RFM) procedure. The combined SOMP-RFM technique has been tested in this work using a pair of remotely sensed scenes collected by the ALI and Hyperion instruments aboard NASA's EO-1 satellite. Although experimental results are very promising, further work is required to fully accommodate all the spectral information available from the multispectral scenes into the registration.

\section{REFERENCES}

[1] L. Brown, "A Survey of Image Registration Techniques" ACM Computing Surveys, vol. 24, no. 4, pp. 326-376, 1992.

[2] B. Zitova and J. Flusser, "Image Registration Methods: A Survey," Image and Vision Computing, vol. 21, pp. 977-1000, 2003.

[3] J. Le Moigne, H. Stone, A. Cole-Rhodes, R. Eastman, P. Jain, K. Johnson, J. Morisette, N. S. Netanyabu, and I. Zavorin, "A Study of the Sensitivity of Automatic Image Registration Algorithms to Initial Conditions," IEEE IGARSS, Anchorage, Alaska, 2004.

[4] A. Plaza, J. Le Moigne, and N. S. Netanyahu, "Automated Image Registration Using Morphological Region of Interest Feature Extraction," IEEE Intl. Workshop on Analysis of Multi-Temporal Images, Biloxi, Missíssippi, pp. 99-103, 2005.

[5] P. Soille, Morphological Image Analysis: Principles and Applications, 2nd ed., Springer, Berlin, 2003.

[6] A. Plaza, P. Martínez, R. Pérez, and J. Plaza, "Dimensionality Reduc tion and Classification of Hyperspectral Image Data Using Sequences of Extended Morphological Transformations," IEEE Trans. Geosci. Remote Sensing, vol. 43, pp. 466-479, 2005.

[7] C.-I. Chang, Hyperspectral imaging: Techniques for spectral detection and classification, Kluwer: New York, 2003.

[8] N. S. Netanyahu, J. Le Moigne, and J. G. Masek, "Georegistration of Landsat Data via Robust Matching of Multiresolution Features," IEEE Trans. Geosci. Remote Sensing, vol. 42, pp. 1586-1600, 2004.

[9] A. Plaza and C.-I Chang, "Impact of Initialization on Design of Endmember Extraction Algorithms," IEEE Trans. Geosci. Remote Sensing, vol. 44, pp. 3397-3407, 2006. 
INTERNATIONAL GEOSCIENCE AND REMOTE SENSING SYMPOSIUM

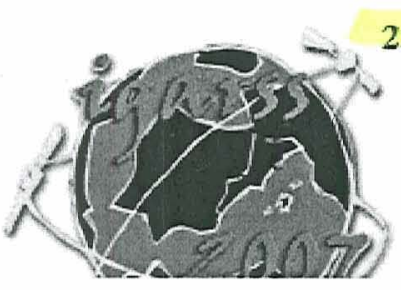

23 - 27 July 2007 - Barcelona, Spain

\section{Sensing And Understanding Our Planet}
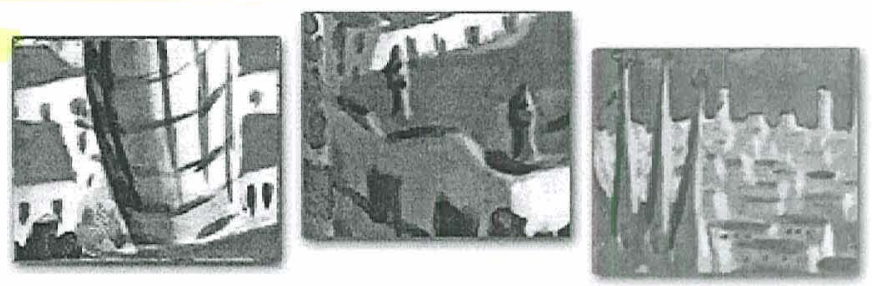

WELCOME

Organizing Committee

Technical Program Committee

Important Dates NEW!

Tutorial Sessions

Author Area

Conference Topics

Registration

Check Abstract Status

Exhibition

Sponsoring

GEOSS Workshop

General Information

Events

Accommodation

Travel Support

Student Prize

Contacts

Preliminary Program

Social Tours NEW!

DOWNLOAD THE

CALL FOR PAPERS
Don't miss the opportunity to experience the flavor of the Mediterranean!. Barcelona, the host city of IGARSS'07, is one of the most emblematic spots of this part of the world. It is Spain's second largest city and the economic, historical and administrative capital of Catalonia, a small country of six million inhabitants, more than a thousand years of history and a culture and language of its own. In Barcelona you will enjoy the unique cultural and artistic ambience of Catalonia, including Modernism, Architecture and South-European culture and cuisine.

The IGARSS' 07 local organizing committee is working hard to set up a high quality technical program as well as social tours. The selected conference venue is the new "Centre de Convencions Internacional de Barcelona" (CCIB), located in one of the newest parts of the city and near the sea front. After a hard day of interesting technical sessions, in just a few minutes you can be admiring the immensity of the sea wandering by the nearby beach. The host hotel is the "AC", located at walking distance of the conference center and offering all the comfort and services of modern hotels. A large selection of other hotels scattered around the city, including modern hotels. A large selection of other hotels scattered around the city, including
the historical part, have also been pre-reserved. For those who prefer seeking their own lodging Barcelona offers a large variety of options, ranging from low price hotels to the most exclusive ones.

The theme of this year symposium -"Sensing and Understanding our Planet"- suggests what remote sensing scientific activity should ultimately achieve: Information gathered by all sensors and techniques must be wisely used mainly to understand our Earth. This will improve prediction of natural disasters or global climate change and provide tools to prevent their consequences Moreover, understanding Earth is needed to devise ways for using natural resources in a sustained way. Goal 7 of the UN millennium goals commits us to "Ensure environmental sustainability" and this is not just a nice sentence. According to the UN report achieving the goal will require greater attention to the plight of the poor, whose day-to-day subsistence is often directly in achieving this goal, as IGARSS is the forum where tools and technological advances for understanding our planet are presented. This is our contribution to the important task of assuring people of all around the world access to resources for their subsistence without endangering the fragile equilibrium of our planet.

The wide variety of topics suggested for the conference range from fundamental concepts to engineering techniques applied to sensor design or data processing and from general applications in all Earth environments to specific on-going or future missions. invite all researches in any aspect related to Geoscience and Remote Sensing, even not specifically listed in the suggested topics, to join us in the most important yearly event of the field.

I look forward to seeing you in Barcelona. Barcelona is waiting for you.

Ignasi Corbella

General Chairman

April, 2006 


\section{INTERNATIONAL GEOSCIENCE AND REMOTE SENSING SYMPOSIUM}

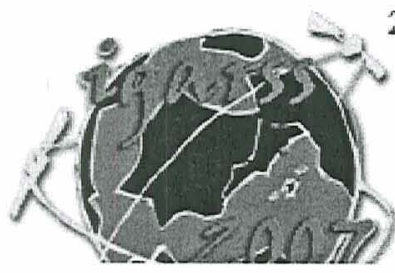

Welcome

Organizing Committee

Technical Program Committee

Important Dates NEW!

Tutorial Sessions

Author Area

Conference Topics

Registration

Check Abstract Status

Exhibition

Sponsoring

GEOSS Workshop

General Information

Events

Accommodation

Travel Support

Student Prize

Contacts

All Sessions and Papers

Social Tours NEW!

DOWNLOAD THE

CALL FOR PAPERS
23 - 27 July 2007 - Barcelona, Spain

\section{Sensing And \\ Understanding Our Planet}

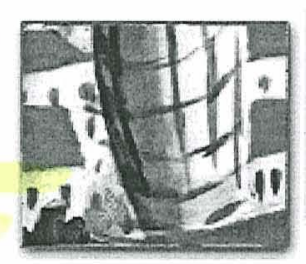

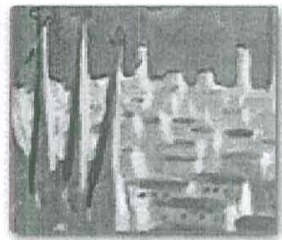

MoEP
Room: Not Assigned

23/07/2007 18:00 - 19:30

Feature Extraction and Reduction

A Feature Selection Algorithm for Class Discrimination Improvement

Claudio De Stefano, Francesco Fontanella, Cristina Marrocco, Gilda Schirinzi

Potential Problems with Using Reconstruction in Morphological Profiles for

Classification of Remote Sensing Images from Urban Areas

Rik Bellens, Leyden Martinez-Fonte, Jonathan Cheung-Wai Chan, Sidharta Gautama, Frank Canters

GIFTS SM EDU Data Processing and Algorithms

Jialin Tian, David G. Johnson, Robert A. Reisse, Michael J. Gazarik

Classification of Landsat TM Image Based on Non-Negative Matrix Factorization Jiamian Ren, Xianchuan Yu, Bixin Hao

Parallel Morphological Feature Extraction for Automatic Registration of Remotely Sensed Images

Antonio Plaza, Jacqueline Le Moigne, Nathan S. Netanyahu

Texture Representation Through Fractal Singularity Spectrum

Daniele Giusto, Valeria Orani

Three-dimensional Tree with Spatial Features Modeling Based on Rules

Zhangang Wang, Dafang Zhuang

Knowledge Centered Earth Observation: Feature Extraction

Amaia De Miguel, Gottfried Schwarz, Mihai Datcu

GTDBSCAN: Leveraging Ground-Truth Geospatial Semantic Annotations for Large Dataset Clustering

Matthew Klaric, Chi-Ren Shyu 